\title{
Construction of risk prediction model of type 2 diabetes mellitus based on logistic regression
}

\author{
Jian $\mathrm{Li}^{1, \#}$, Qin Huang ${ }^{2}$, Minghua Dong ${ }^{3, \#}$, Wei Qiu ${ }^{3}$, Lixia Jiang ${ }^{4}$, Xiaoting Luo ${ }^{5}$, Zhengchun Huang ${ }^{1}$, \\ Shuiqin Chen ${ }^{5}$, Qinfeng Wu ${ }^{3}$, Lu Ou-Yang ${ }^{3}$, Qin Wu ${ }^{4}$, Lihua Liu ${ }^{5}$ and Shumei Li ${ }^{3}$, a \\ ${ }^{1}$ Department of Anatomy Gannan Medical College, Ganzhou, Jiangxi, China \\ ${ }^{2}$ Department of General Medicine, St.Michael hospital, Shanghai, China \\ ${ }^{3}$ Department of Epidemiology in Preventive Medicine, Gannan Medical College, Ganzhou, Jiangxi, China \\ ${ }^{4}$ Department of Clinical laboratory of 1 'th affiliate hospital, Gannan Medical College, Ganzhou, Jiangxi, China \\ ${ }^{5}$ Department of Biochemistry and Molecular Biology, Gannan Medical College, Ganzhou, Jiangxi, China
}

\begin{abstract}
Objective: to construct multi factor prediction model for the individual risk of T2DM, and to explore new ideas for early warning, prevention and personalized health services for T2DM. Methods: using logistic regression techniques to screen the risk factors for T2DM and construct the risk prediction model of T2DM. Results: Male's risk prediction model logistic regression equation: $\operatorname{logit}(\mathrm{P})=\mathrm{BMI} \times 0.735+$ vegetables $\times(-0.671)+$ age $\times 0.838+$ diastolic pressure $\times 0.296+$ physical activity $\times(-2.287)+$ sleep $\times(-0.009)+$ smoking $\times 0.214$; Female's risk prediction model logistic regression equation: $\operatorname{logit}(\mathrm{P})=\mathrm{BMI} \times 1.979+$ vegetables $\times(-0.292)$ + age $\times 1.355+$ diastolic pressure $\times 0.522+$ physical activity $\times(-2.287)+$ sleep $\times(-0.010)$. The area under the ROC curve of male was 0.83 , the sensitivity was 0.72 , the specificity was 0.86 , the area under the ROC curve of female was 0.84 , the sensitivity was 0.75 , the specificity was 0.90. Conclusion: This study model data is from a compared study of nested case, the risk prediction model has been established by using the more mature logistic regression techniques, and the model is higher predictive sensitivity, specificity and stability.
\end{abstract}

\section{Introduction}

With the rapid development of social economy, people's life style and dietary structure have changed and population aging intensified, diabetes prevalence rate rose rapidly, especially in our country [1], it has brought heavy economic burden to society and family. In recent years, foreign countries have tended to use a risk assessment tool to the risk of type 2 diabetes mellitus(T2DM) for prediction and risk score, for finding the early identification of high-risk groups, to control risk factors by carrying out the health education and lifestyle intervention, and then reduce the T2DM [2]. Some domestic scholars also constructed T2DM risk model with risk factors scoring method and OR data score and so on, to predict high-risk T2DM; and, in this aspect, they did some propaganda and education to make people accept the T2DM risk individual prediction model [3-4] in the identification of high-risk

\footnotetext{
${ }^{a}$ Corresponding author: Shumei Li, gnyxylsm@163.com. Phone: 008615083787928.

"These authors contributed equally to this study and share first authorship.

This study was supported by scientific fund from National Natural Science Fund in China (No.81360445), Science and technology support program of Jiangxi Province (NO.20132BBG70086).
} 
groups gradually .

\section{Research design and program}

\subsection{Research objects}

Researchers chose the sample from T2DM baseline survey for the project study in 19 districts and counties in Ganzhou City in 2009. The study subject is a total of 8086 copies of data, age 35-64 years old. The baseline survey and follow-up data content include general demographic characteristics, smoking and drinking history, personal health history, diabetes family history, physical activity and physical exercise and so on; human body measurements included height, weight, BMI, waist circumference, hip circumference, waist hip ratio (WHR), blood pressure, lung capacity and so on ; biochemical metabolic index included fasting blood glucose (FBG), total cholesterol (TC), triglyceride (TG), high density lipoprotein cholesterol (HDL-C), low density lipoprotein cholesterol (LDL-C), blood uric acid (UA) (save all to MSSQL database).

\subsection{Research methods}

Researchers randomly selected 2 / 3 samples (5391persons) from MSSQL database as the training group, the pre-selected variables is age, sex, BMI, waist circumference and waist to hip ratio (WHR), blood pressure (BP), T2DM family history, physical activity, smoking and drinking, FBG, TC, TG, HDL-C, LDL-C; and then made single factor analysis for the pre-seleced variables . Multivariate analysis mainly adopted mature logistic regression model, as to whether diabetes as the dependent variable (diabetic patient with the value 1 , non diabetic patient with the value 0 ), other variables (suspected risk factor variables) as independent variables were multivariate logistic regression, $\operatorname{logistic}(\mathrm{p})=\ln \left(\frac{p}{1-p}\right)$, to construct the T2DM risk prediction model. In order to increase the prediction accuracy, prediction model of gender were fitted.Another $1 / 3$ sample (2695 people) in the study cohort was used as the test group, the area under the receiver operating characteristic curve (ROC) was used to analyze the sensitivity and specificity of the model, to evaluate the predictive effect of the model .

\section{Results}

\subsection{Relationship between age, sex and T2DM}

Research data showed the prevalence of T2DM was $7.7 \%$, with group of every 5 years, people will be divided into 35-, 40-, 45-, 50-, 55-, 60-64, T2DM prevalence was compared between six groups by gender, the differences of T2DM prevalence between each group were not statistically significant $(p>0.05)$.(see Table 1$)$.

Table 1. Different gender and age in T2DM prevalence rate.

\begin{tabular}{lllllll}
\hline \multirow{2}{*}{ age(year) } & \multicolumn{3}{c}{ male } & \multicolumn{3}{c}{ female } \\
\cline { 2 - 7 } & $\mathrm{n}$ & $\mathrm{T} 2 \mathrm{DM}$ & $\%$ & $\mathrm{n}$ & $\mathrm{T} 2 \mathrm{DM}$ & $\%$ \\
\cline { 2 - 7 } $35-$ & 288 & 8 & 2.8 & 368 & 13 & 3.5 \\
$40-$ & 362 & 20 & 5.5 & 439 & 12 & 2.7 \\
$45-$ & 375 & 22 & 5.9 & 402 & 21 & 5.2 \\
$50-$ & 273 & 22 & 8.1 & 395 & 28 & 7.1 \\
$55-$ & 390 & 50 & 12.8 & 475 & 41 & 8.6 \\
$60-64$ & 378 & 53 & 14.0 & 363 & 57 & 15.7 \\
total & 2066 & 175 & 8.5 & 2442 & 172 & 7.0 \\
\hline
\end{tabular}




\subsection{Relationship between the body mass index $\left(\mathrm{kg} / \mathrm{M}^{2}\right)$ and T2DM}

Body mass index (BMI) were divided into three groups: the normal group (18.5-23.9),T2DM prevalence was $6.7 \%(88 / 1315)$; hyper height group( $\geq 24.0)$, T2DM prevalence was $9.6 \%(86 / 897)$; obesity group $(\geq 28)$, T2DM prevalence was $14.9 \%(35 / 235)$. The three groups were compared with the increase of BMI and the prevalence of T2DM, the difference was statistically significant $\left(x^{2}=19.15, p<0.001\right)$.

BMI group by gender: Male, T2DM prevalence of normal group was 7.3\% (42/578);T2DM prevalence of overweight group was 9.9\% (41/414); T2DM prevalence of obesity group was $17.2 \%$ (16/93); There are differences between these groups and these differences were statistical significant $\left(x^{2}=10.03, p<0.05\right)$. Female, T2DM prevalence of the normal group was $6.2 \%(46 / 737)$; T2DM prevalence of overweight group was 9.3\% (45/483); T2DM prevalence of obesity group was $13.4 \%$ (19/142); There are differences between these groups and these differences was statistical significant $\left(x^{2}=9.72, p<0.05\right)$. (see Table 2$)$.

Table 2. T2DM prevalence rate of different BMI In different gender.

\begin{tabular}{lllllll}
\hline BMI & \multicolumn{2}{c}{ male } & \multicolumn{3}{c}{ female } \\
\cline { 2 - 7 }$\left(\mathrm{kg} / \mathrm{m}^{2}\right)$ & $\mathrm{n}$ & $\mathrm{T} 2 \mathrm{DM}$ & $\%$ & $\mathrm{n}$ & $\mathrm{T} 2 \mathrm{DM}$ & $\%$ \\
\cline { 2 - 7 } $18.5-23.9$ & 578 & 42 & 7.3 & 737 & 46 & 6.2 \\
$\geq 24.0$ & 414 & 41 & 9.9 & 483 & 45 & 9.3 \\
$\geq 28$ & 93 & 16 & 17.2 & 142 & 19 & 13.4 \\
total & 1085 & 99 & 9.1 & 1362 & 110 & 8.1 \\
\hline
\end{tabular}

\subsection{Relationship between waist circumference and waist to hip ratio of T2DM}

Waist samples were divided into two groups: normal group (male $<85 \mathrm{~cm}$, female $<80 \mathrm{~cm}$ ), T2DM prevalence was $5.7 \%$ (79/1377); abnormal group (male $\geq 85 \mathrm{~cm}$, the female $\geq 80 \mathrm{~cm}$ ), T2DM prevalence was $12.2 \%$ (129/1060). Waist circumference increased, the prevalence of T2DM increased, the difference was statistically significant $\left(x^{2}=31.74, p<0.001\right)$. Male, T2DM prevalence of normal group was 7.0\% (45/640); T2DM prevalence of abnormal group was $12.2 \%(54 / 443)$; There are differences between these groups and these differences were statistical significant $\left(x^{2}=8.39, p<0.05\right)$. Female, T2DM prevalence of the normal group was 4.6\% (34/737); T2DM prevalence of abnormal group was $12.2 \%(75 / 617)$; There are differences between these groups and these differences was statistical significant $(p<0.05)$. (see Table 3$)$.

Table 3. Relationship between waist circumference and T2DM

\begin{tabular}{lllllll}
\hline waist $(\mathrm{cm})$ & \multicolumn{3}{c}{ male } & \multicolumn{3}{c}{ female } \\
\cline { 2 - 7 } & $\mathrm{n}$ & T2DM & $\%$ & $\mathrm{n}$ & $\mathrm{T} 2 \mathrm{DM}$ & $\%$ \\
\cline { 2 - 7 } male $<85$, female $<80$ & 640 & 45 & 7.0 & 737 & 34 & 4.6 \\
male $\geq 85$, female $\geq 80$ & 443 & 54 & 12.2 & 617 & 75 & 12.2 \\
total & 1083 & 99 & 9.1 & 1354 & 109 & 8.1 \\
\hline
\end{tabular}

The samples was divided into three groups by the ratio of waist to hip $(\mathrm{W}-\mathrm{H})$, the low group (male $\mathrm{WH}<0.82$, female $\mathrm{WH}<0.78$ ), the prevalence of $\mathrm{T} 2 \mathrm{DM}$ was $3.9 \%(16 / 410)$; the normal group (male $0.82 \leq \mathrm{WH}<0.91$, female $0.78 \leq \mathrm{WH}<0.87)$, the prevalence of $\mathrm{T} 2 \mathrm{DM}$ was $6.6 \%(83 / 1267)$; the high group (male $\mathrm{WH} \geq 0.91$, female $\geq 0.87$ ), the prevalence of $\mathrm{T} 2 \mathrm{DM}$ was $14.2 \%$, 107/754). Compared with the three groups between male and female, WH increased, the prevalence of T2DM increased, the difference was statistically significant $(p<0.05)$.(see Table 4$)$. 
Table 4. Waist hip ratio and the prevalence of T2DM relations.

\begin{tabular}{lllllll}
\hline W-H & \multicolumn{3}{c}{ male } & \multicolumn{3}{c}{ female } \\
\cline { 2 - 7 } & $\mathrm{n}$ & $\mathrm{T} 2 \mathrm{DM}$ & $\%$ & $\mathrm{n}$ & $\mathrm{T} 2 \mathrm{DM}$ & $\%$ \\
\cline { 2 - 7 } male $<0.82$, female $<0.78$ & 207 & 13 & 6.3 & 203 & 3 & 1.5 \\
male $0.82-0.91$, female $0.78-0.87$ & 588 & 44 & 7.5 & 679 & 39 & 5.7 \\
male $\geq 0.91$, female $\geq 0.87$ & 286 & 40 & 14.0 & 468 & 67 & 14.3 \\
total & 1081 & 97 & 9.0 & 1350 & 109 & 8.1 \\
\hline
\end{tabular}

\subsection{Relationship between blood pressure and the prevalence of T2DM}

The samples were divided into two groups by the blood pressure, the normal group (systolic blood pressure $\mathrm{SBP}<140 \mathrm{mmHg}$ and diastolic blood pressure $\mathrm{DBP}<90 \mathrm{mmHg}$ ), and hypertension group (SBP $\geq 140 \mathrm{mmHg}, \mathrm{DBP} \geq 90 \mathrm{mmHg}$.

Normal group of Systolic blood pressure(SBP)'s T2DM prevalence was $6.9 \%(132 / 1920)$. Hypertension group of SBP 's T2DM prevalence was $14.8 \%(76 / 512)$; the prevalence of T2DM in hypertension group increased, and the difference is statistically significant $(p<0.05)$. The normal group of diastolic blood pressure(DBP)'s T2DM prevalence was 7.6\% (141/1853) ; hypertension group of DBMS's T2DM prevalence was $11.7 \%$ (67/575), the prevalence of hypertension group was different in T2DM,for male, the difference has no statistical significance ( $p>0.05)$;for female, the difference has statistical significance $(p<0.05)$. (see Table 5,6).

Table 5. SBP and T2DM prevalence rate.

\begin{tabular}{lllllll}
\hline SBP & \multicolumn{3}{c}{ male } & \multicolumn{3}{c}{ female } \\
\cline { 2 - 7 }$(\mathrm{mmHg})$ & $\mathrm{n}$ & $\mathrm{T} 2 \mathrm{DM}$ & $\%$ & $\mathrm{n}$ & $\mathrm{T} 2 \mathrm{DM}$ & $\%$ \\
\cline { 2 - 7 }$<140$ & 842 & 63 & 7.5 & 1078 & 69 & 6.4 \\
$\geq 140$ & 237 & 35 & 14.8 & 275 & 41 & 14.9 \\
total & 1079 & 98 & 9.1 & 1353 & 110 & 8.1 \\
\hline
\end{tabular}

Table 6. DBP and T2DM prevalence rate.

\begin{tabular}{lllllll}
\hline DBP & \multicolumn{3}{c}{ male } & \multicolumn{3}{c}{ female } \\
\cline { 2 - 7 }$(\mathrm{mmHg})$ & $\mathrm{n}$ & $\mathrm{T} 2 \mathrm{DM}$ & $\%$ & $\mathrm{n}$ & $\mathrm{T} 2 \mathrm{DM}$ & $\%$ \\
\cline { 2 - 7 } & 805 & 66 & 8.2 & 1048 & 75 & 7.2 \\
$\geq 90$ & 273 & 32 & 11.7 & 302 & 35 & 11.6 \\
total & 1078 & 98 & 9.1 & 1350 & 110 & 8.1 \\
\hline
\end{tabular}

\subsection{Relationship between total cholesterol (TC) and T2DM}

The total cholesterol index sample was divided into three groups: the low group , TC $<5.18 \mathrm{mmol} / \mathrm{L}$, T2DM prevalence was $6.2 \%(130 / 2105)$; intermediate group , $5.18 \mathrm{mmol} / \mathrm{L} \leq \mathrm{TC}<6.22 \mathrm{mmol} / \mathrm{L}, \mathrm{T} 2 \mathrm{DM}$ prevalence was $10.2 \%(80 / 783)$; high group , $\mathrm{TC} \geq 6.22 \mathrm{mmol} / \mathrm{L}, \mathrm{T} 2 \mathrm{DM}$ prevalence was $18.6 \%$ (49/264). The prevalence of hypertension group was different in T2DM:for male and female, the difference of group has no statistical significance $(p>0.05)$; for whole sample the difference has statistical significance $(p<0.05)$.(see Table 7)

Table 7. Regardless of gender, the relationship between cholesterol and prevalence rate of T2DM.

\begin{tabular}{lllllll}
\hline TC & \multicolumn{3}{c}{ male } & \multicolumn{3}{c}{ female } \\
\cline { 2 - 7 }$(\mathrm{mmol} / \mathrm{L})$ & $\mathrm{n}$ & $\mathrm{T} 2 \mathrm{DM}$ & $\%$ & $\mathrm{n}$ & $\mathrm{T} 2 \mathrm{DM}$ & $\%$ \\
\cline { 2 - 7 }$<5.18$ & 927 & 74 & 8.0 & 1178 & 56 & 4.8 \\
$5.18-6.22$ & 328 & 33 & 10.1 & 455 & 47 & 10.3 \\
$\geq 6.22$ & 101 & 14 & 13.9 & 163 & 35 & 21.5 \\
total & 1356 & 121 & 8.9 & 1350 & 138 & 7.7 \\
\hline
\end{tabular}




\subsection{Relationship between triglyceride (TG) and T2DM}

Triglyceride sample was divided into three groups: low group , $\mathrm{TG}<1.70 \mathrm{mmol} / \mathrm{L}$, the prevalence of T2DM was $6.0 \%(132 / 2209)$; intermediate group, $1.70 \mathrm{mmol} / \mathrm{L} \leq \mathrm{TG}<2.26 \mathrm{mmol} / \mathrm{L}$, the prevalence of $\mathrm{T} 2 \mathrm{DM}$ is $9.5 \%(46 / 482)$; high group , $\mathrm{TG} \geq 2.26 \mathrm{mmol} / \mathrm{L})$, the prevalence of $\mathrm{T} 2 \mathrm{DM}$ was $17.9 \%$ (82/459). Compared with the three groups between male and female, TG increased, the prevalence of T2DM increased, the difference was statistically significant $(p<0.001)$.(see Table 8$)$.

Table 8. Relationship between triglyceride index and the prevalence rate of T2DM.

\begin{tabular}{lllllll}
\hline TG & \multicolumn{3}{c}{ male } & & \multicolumn{3}{c}{ female } \\
\cline { 2 - 7 }$(\mathrm{mmol} / \mathrm{L})$ & $\mathrm{n}$ & $\mathrm{T} 2 \mathrm{DM}$ & $\%$ & $\mathrm{n}$ & $\mathrm{T} 2 \mathrm{DM}$ & $\%$ \\
\cline { 2 - 7 }$<1.70$ & 918 & 64 & 7.0 & 1291 & 68 & 5.3 \\
$1.70-2.26$ & 210 & 18 & 8.1 & 272 & 28 & 10.3 \\
$\geq 2.26$ & 225 & 39 & 17.3 & 234 & 43 & 18.4 \\
total & 1353 & 121 & 8.9 & 1797 & 139 & 7.7 \\
\hline
\end{tabular}

\subsection{Relationship between smoking and T2DM}

The subjects were divided into two groups, low exposure group (no smoking) and high exposure group (smoking): there was no significant difference in the prevalence of low exposure group and high exposure group $\left(x^{2}=0.831, p>0.05\right)$.

\subsection{The Relationship between dietary factors and T2DM}

Compared the people who often eat coarse grain with the people who don't eat the coarse grain, male's OR was $0.41,95 \%$ confidence interval $(0.25,0.67)$, The differences of prevalence have statistical significance $\left(x^{2}=13.12 . p<0.001\right)$, coarse grain is the protective factor; Female's OR is 0.73 , $95 \%$ confidence interval for $(0.47,1.50)$, the differences of prevalence have statistical significance $\left(x^{2}=13.18, p<0.05\right)$; coarse grains is the protective factor.

Compared the people who often edible meat with the people who don't eat meat,male's OR is 2.37 , $95 \%$ confidence interval $(1.46,3.84)$, the differences in prevalence have statistical significance $\left(x^{2}=12.92, p<0.001\right)$, meat is the risk factor ; The difference of female prevalence have no statistical significance $\left(x^{2}=0.26, p>0.05\right)$.

Compared the people who often edible vegetable with the people who don't edible the vegetables, male's OR is $0.59,95 \%$ confidence interval for $(0.37,0.93)$, the differences in prevalence have statistical significance $\left(x^{2}=5.16, p<0.05\right)$,vegetable is the protective factor; Female's OR is $0.50,95 \%$ confidence interval for $(0.32,0.77)$, the differences in prevalence have statistical significance $\left(x^{2}=9.92\right.$, $p<0.05)$; vegetable is the protective factor.

\subsection{Multiple factors analysis of logistic regression}

The analysis of Significant predictive variables had showed : male subjects in the study taking BMI, age, diastolic blood pressure, smoking as the risk factor, and vegetable intake, physical activity, sleep as the protective factor $(\beta<0)$, and risk prediction model regression equation logit $(\mathrm{P})=\mathrm{BMI} \times 0.735+$ vegetables $\times(-0.671)+$ age $\times 0.838+$ diastolic pressure $\times 0.296+$ physical activity $\times(-2.287)$ sleep $\times(-$ $0.009)+$ smoking $\times 0.214$. The female subjects in the study taking BMI, age, diastolic pressure as the risk factor, and vegetable intake, physical activity, sleep as the protective factor, risk prediction model regression equation logit $(\mathrm{P})=\mathrm{BMI} \times 1.979+$ vegetables $\times(-0.292)+$ age $\times 1.355+$ diastolic pressure $\times$ $0.522+$ physical activity $\times(-2.287)+$ sleep $\times(-0.010)$. (see Table 9,10$)$ 
Table 9. The Results of logistic regression analysis of male relative indexes.

\begin{tabular}{lllll}
\hline Variable beta & $\beta$ & S.E & $x^{2}$ & P \\
\hline Body mass index & 0.735 & 0.905 & 0.659 & 0.417 \\
Vegetable & -0.671 & 0.292 & 5.291 & 0.021 \\
Age & 0.838 & 0.497 & 2.843 & 0.092 \\
Diastolic blood pressure & 0.296 & 0.303 & 0.956 & 0.328 \\
Physical activity & -2.287 & 0.106 & 465.380 & 0.000 \\
Sleep & -0.009 & 0.166 & 0.003 & 0.956 \\
Smoking & 0.214 & 0.235 & 0.828 & 0.363 \\
\hline
\end{tabular}

Table 10.The Results of logistic regression analysis of female related indexes.

\begin{tabular}{lllll}
\hline Variable beta & $\beta$ & S.E & $x^{2}$ & $\mathrm{P}$ \\
\hline Body mass index & 1.979 & 1.056 & 3.512 & 0.061 \\
Vegetable & -0.292 & 0.316 & 0.855 & 0.355 \\
Age & 1.355 & 0.588 & 5.318 & 0.021 \\
Diastolic blood pressure & 0.522 & 0.333 & 2.460 & 0.117 \\
Physical activity & -2.287 & 0.106 & 465.380 & 0.000 \\
sleep & -0.010 & 0.123 & 0.007 & 0.956 \\
\hline
\end{tabular}

\subsection{The Internal authenticity of the model and its evaluation}

Male' s area under the ROC curve area was 0.83 , sensitivity was 0.72 , specificity was 0.86 ; female's area under ROC curve was 0.84 , sensitivity was 0.75 , specificity was 0.90 , two sets of data showed that model had a high predictive value.(see Figure 1).

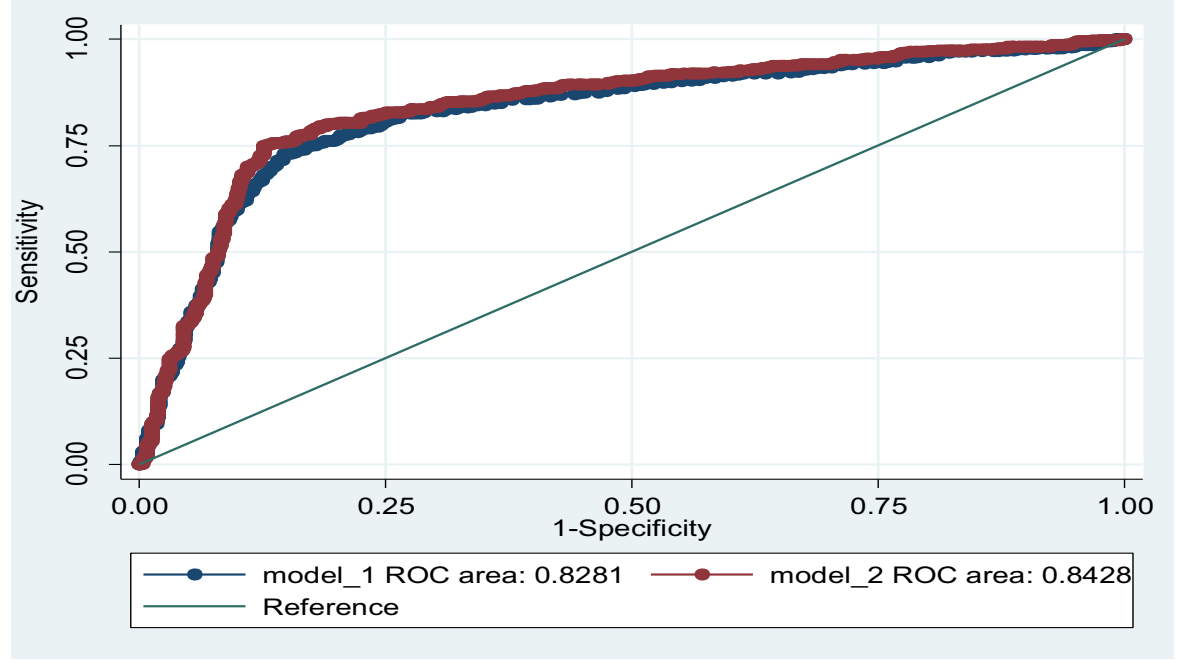

Figure 1. Sensitivity and specificity of the model.

\section{Discussion}

The modeling data was from compared study of prophase nested case, and used the less error and more mature logistic regression techniques in theory to deal with the variables. The risk model that had been established on this base has higher sensitivity, specificity and stability. The development of T2DM risk model started late. Since 2003, some type 2 diabetes risk assessment tools had been 
gradually developed and applicated in foreign country [5-9], but due to the difference in race, behavior and lifestyle, diet, medical and health services, economic and cultural factors, foreign risk assessment tool was not fully applicable to Asian and / or Chinese [10-11].Therefore, since 2006, scholars in our country [12-14] have concluded that the main risk factors of diabetes mellitus and its relative risk to establish evaluation methods and evaluation model of adult individual diabetes risk in our country, on the basis of epidemiological survey of diabetes ,by risk factors scoring method, disease risk score, the analysis of diabetes risk factors and prevalence data. These models mostly based on the compared study cases, prospective study was insufficient; Our study team has fully considered the conditions which required for robust risk model, include stable and representative large samples, a prospective observation, multivariate estimation and mature computer technology platform .But the research is still in initial stage with many remaining problems: the first is the study did not exclude the influence of the linear of predictive variables in model to the stability of the model; researchers only evaluate the predictive model by the similar people, part of the data is not so complete; We will evaluate and verificate this model by screening return visitors in the latter part of the study .

\section{References}

1. Yang WY, Lu JM, Weng Jp, N Engl J Med ,Prevalence of diabetes among men and women in China,362,1090-1101(2010).

2. Douglas N, Rohini M,Tom D, BMJ,Risk models and scores for type 2 diabetes: systematic review, 343, d7163(2011).

3. Mann DM, Bertoni AG, Shimbo D, Am J Epidemiol, Comparative Validity of 3 Diabetes Mellitus Risk prediction Scoring Models in a Multiethnic US Cohort The Multi-Ethnic Study of Atherosclerosis, 171,980-988(2010).

4. Almeda-Valdes p, Cuevas-Ramos D, Mehta R,Curr Diabetes Rev,UKpDS Risk Engine, decode and diabetespHD models for the estimation of cardiovascular risk in patients with diabetes, 6,18(2010).

5. Buijsse B, Simmons RK, Griffin SJ, Schulze MB,Epidemiol Rev, Risk Assessment Tools for Identifying Individuals at Risk of Developing Type 2 Diabetes, 33,46-62(2011).

6. Lindström J, Tuomilehto J, Diabetes Care,The diabetes risk score: a practical tool to predict type 2 diabetes risk,26,725-731(2003).

7. Schulze MB, Weikert C, pischon T, Diabetes Care, Use of multiple metabolic and genetic markers to improve the prediction of type 2 diabetes: the EpIC-potsdam Study,32,2116-2119(2009).

8. Glmer,Charlotte,Carstensen,Bendix,Sandbk,Annelli,Lauritzen,Torsten,Jrgensen,Torben,BorchJohnsen,Knut, Diabetes Care ,A Danish Diabetes Risk Score for Targeted Screening ,3,727733(2004).

9. Heikes KE, Eddy DM, Arondekar B, Diabetes Care, Diabetes Risk Calculator: a simple tool for detecting undiagnosed diabetes and pre-diabetes,31,1040-1045(2008).

10. Liu M,Pan CY, Jing MM, Su HY,Lu JM, Chinese Journal of diabetes, Evaluation of diabetes risk score in the evaluation of new onset diabetes,17,201 -204(2009).

11. 12.Calvin Woon-Loong Chin, Elia n Hui San Chia, Stefan Ma, Derrick Heng, Maud rene Tan, Jean ette Lee, E Shyong Tai, Agus Salim, BMC,The ARIC predictive model reliably predicted risk of type II diabetes in Asian populations, 1 -13(2012).

12. Li XY, Li R, Zhang SN, Chinese public health, Effect evaluation of different screening methods for asymptomatic diabetes,22,687-689(2006).

13. Wu HY, Pan P, He Y, Chinese Journal of health management, The risk assessment method for adults with diabetes in China,1,95-98(2007).

14. Zhang L, Shi K, Yi D, Chongqing medical, Study on risk assessment of diabetes in community residents in Chongqing City,40,1885-1888(2011). 